\title{
Experimental Investigation on the Anisotropic Tensorial Eddy Viscosity Model for Turbulence Flow
}

\author{
Zhu Zhenwei * and Li Haixia \\ School of Energy Science and Engineering of Henan Polytechnic University, Jiaozuo Henan \\ 454000, China
}

Email: zhuzhenwei22@sina.com

\begin{abstract}
Hot film anemometer was used to perform the experimental investigation on the tensorial eddy viscosity model in a water channel. The time sequence of instantaneous wall-normal velocity components at different locations in water channel have been finely measured by constant temperature anemometer model 1050 and $\mathrm{X}$ shape water probe. Experimental research on the model of anisotropic tensorial eddy viscosity was undertaken using the experimental dataset. The spatial distribution laws for each components of eddy viscosity tensor were presented for incompressible turbulent flow.
\end{abstract}

Keywords: Turbulent flow, Reynolds stress, Tensorial eddy viscosity model, Hot film anemometer.

\section{INTRODUCTION}

A time averaging assumption of turbulent flow was proposed by Reynolds in 1895 . In this assumption, the irregular turbulent flow was decomposed into two parts: the mean part and the fluctuation part. Using the Reynolds decomposition, we can substitute into the Navier-Stokes equations and obtain the averaging Navier-Stokes equations (or Reynolds-averaged equations). A crucial differences between Navier-Stokes equations and Reynolds-averaged equations is about the additional Reynolds stresses term which is a second-order tensor including fluctuation velocity and then make the Reynolds- averaged equations unclosed, this is widely known the closure problem in turbulent flow. In order to resolve the problem of unclosure of Reynolds-stress equations, many hypothesis about Reynolds stress term were introduced on the basis of the theory and the practice, which made the establishment of the turbulence modeling theory. Nowsday, the turbulence modeling theory has become the most effective ways to prediction of turbulence problems and widely be used in engineering[1] [2].

Eddy-viscosity model is used widely in the theory of turbulence. Eddy-viscosity hypothesis-introduced by Boussinesq in 1877-is mathematically analogous to the stress-rate-of-strain relation for a Newtonian fluid, it assume that Reynolds stresses are proportion to the mean rate-ofstrain. Boussinesq[3] established direct relations of Reynolds stress tensor $-\rho \overline{u_{i}^{\prime} u_{j}^{\prime}}$ and the mean rate-of-strain tensor $S_{i j}=\left(\partial \overline{u_{i}} / \partial x_{j}+\partial \overline{u_{j}} / \partial x_{i}\right) / 2$ through introducing scalar eddy viscosity coefficient, and then resolved the closed problem of Reynolds-stress equations: $-\rho \overline{u_{i}^{\prime} u_{j}^{\prime}}=\mu_{T}\left(\frac{\partial \overline{u_{i}}}{\partial x_{j}}+\frac{\partial \overline{u_{j}}}{\partial x_{i}}\right)$

Here, $\mu_{T}$ is mean eddy viscosity coefficient , $\mathrm{i}, \mathrm{j}=1,2,3$.

Eddy-viscosity model introduced by Boussinesq is the theoretical foundation of one-equation models and twoequation models. The $k-\varepsilon$ two-equation model adopt the modified form of Eddy-viscosity model[4]:

$-\rho \overline{u_{i}^{\prime} u_{j}^{\prime}}+\frac{2}{3} k \delta_{i j}=2 \mu_{T} S_{i j}$

Here, $k$ is mean turbulent kinetic energy, and $k=\rho \overline{u_{i}^{\prime} u_{\mathrm{i}}^{\prime}} / 2$.

Eddy-viscosity hypothesis is analogous to the stress-rateof-strain relation for a Newtonian fluid base on homogeneous isotropic turbulence motion, it gives the equation as follows:

$\tau_{i j}=\mu\left(\frac{\partial u_{i}}{\partial x_{\mathrm{j}}}+\frac{\partial u_{j}}{\partial x_{i}}\right)-\left(p+\frac{2}{3} \nabla \bullet \vec{v}\right) \delta_{i j}$

Here, $\tau_{i j}$ is the viscous stress, $p$ is fluid pressure, $\nabla \bullet \vec{v}$ is the divergence of fluid velocity, and $\mu$ is the viscosity coefficient of fluid molecules.

Eq.(3) is established base on the hypothesis of homogeneous isotropic turbulence motion. It's appropriate to the gas and the simple liquid (e.g. water). But for anisotropic 
turbulence motion, the general expression of Eddy-viscosity model should be:

$\tau_{i j}=\mu_{i j k l}\left(\frac{\partial u_{k}}{\partial x_{l}}+\frac{\partial u_{l}}{\partial x_{k}}\right)$

That is, $\tau_{i j}$ should be the linear function of velocity strain rate tensor, $\mu_{i j k l}$, viscosity coefficient, should fourth order tensor[5].

According to molecular motion theory, gas viscosity is thought to be caused by intermolecular momentum exchange induced by random thermal motion of gas molecules. When molecule A collides with molecule B, molecule A make molecule B decelerated; in contrast, molecule B will make molecule A accelerated. From point of macroscopic view, gas has its viscosity and viscous stress $\tau_{i j}$ comes into being.

Random thermal motion of gas molecules is so frequently that the probability in all direction can be thought to be equal. For this reason, viscous stress of gas is thought to be isotropic macroscopically, its constitutive equation of isotropy is reasonable [6] .

The basic element of turbulent motion is eddy which length scale is far larger than any molecular length scale. Reynolds stress of turbulence is caused by fluctuation of large scale eddies [7]. When an eddy collides with other eddies, momentum exchanges will occur among the large scale eddies, this is the forces we called Reynolds stress $-\rho \overline{u_{i}^{\prime} u_{j}^{\prime}}$. In analogy with the constitutive relation of Newtonian fluid, the relation between Reynolds stress and mean rate-of-strain is established by introducing eddy viscosity coefficient $\mu_{T}$ instead of $\mu$.But the physics property of coefficient $\mu_{T}$ differs from coefficient $\mu$. Firstly, viscosity coefficient $\mu$ belongs to fluid physical property without considering whether the flow is in the state of laminar or turbulence, But eddy viscosity coefficient $\mu_{T}$ doesn't belong to physical property of the fluid, it relies on turbulence flow state. The problem of Eddy-viscosity model is to find out the relation between parameters of flow field and eddy viscosity coefficient $\mu_{T}$. Secondly, Reynolds stress is anisotropy, but not the molecular viscous stress. The anisotropy of Reynolds stress in turbulent flow relies mainly on large scale eddies $[8,9]$. The Boussinesq eddy viscosity model was established on the hypothesis that Reynolds stress is isotropy, this will lead to the difference between Boussinesq eddy viscosity model and actually Reynolds stress. Wang Zhen-dong [10,11] proposed a tensorial eddy viscosity model to reflect the anisotropy of Reynolds stress in which the eddy viscosity coefficient is the disymmetry fourth-order tensor. Using this model will be more reasonable than before. In this model, Reynolds stress are written as follows:

$-\rho \overline{u_{i} u_{j}}=\varepsilon_{i j k l}\left[\frac{1}{2}\left(\frac{\partial u_{k}}{\partial x_{l}}+\frac{\partial u_{l}}{\partial x_{k}}\right)\right]$

considering the conservation of turbulent kinetic energy according to Eq.(2), thus, tensorial eddy viscosity model is given by be: $-\rho \overline{u_{i} u_{j}}=\varepsilon_{i j k l}\left[\frac{1}{2}\left(\frac{\partial u_{k}}{\partial x_{l}}+\frac{\partial u_{l}}{\partial x_{k}}\right)\right]-\frac{2}{3} \delta_{i j} k$

For the flow of two-dimensional incompressible turbulence, three components of the Reynolds stress and three components of the strain rate tensor are involved in Reynolds- averaged equations. Under this condition, there are six independent components of the eddy viscosity tensors $\varepsilon_{i j k l}$, each component can be calculated as follows:

$$
\begin{aligned}
& \left(\varepsilon_{i j 11}, \varepsilon_{i j 12}, \varepsilon_{i j 21}, \varepsilon_{i j 22}\right)= \\
& \frac{-\rho \overline{u_{i} u_{j}}+\frac{2}{3} \delta_{i j} k}{\|\beta\|^{2}}\left[\frac{\partial u_{1}}{\partial x_{1}}, \frac{1}{2}\left[\frac{\partial \overline{u_{1}}}{\partial x_{2}}+\frac{\partial \overline{u_{2}}}{\partial x_{1}}\right], \frac{1}{2}\left[\frac{\partial \overline{u_{1}}}{\partial x_{2}}+\frac{\partial \overline{u_{2}}}{\partial x_{1}}\right], \frac{\partial u_{2}}{\partial x_{2}}\right]^{(7)} \\
& \text { Here, } i, j=1,2 ;\|\beta\|^{2}=\left[\frac{\partial u_{1}}{\partial x_{1}}\right]^{2}+\frac{1}{2}\left[\frac{\partial \overline{u_{1}}}{\partial x_{2}}+\frac{\partial \overline{u_{2}}}{\partial x_{1}}\right]^{2}+\left[\frac{\partial u_{2}}{\partial x_{2}}\right]^{2} .
\end{aligned}
$$

Our objective of the present article is to measure the instantaneous velocity time sequence at different streamwise and vertical locations in a water channel by using the hot film anemometer model 1050, and analyze the spatial distribution pattern of the components of anisotropy eddy viscosity tensor by the data from the experiment in order to improve more reasonable turbulence model.

\section{EXPERIMENTAL FACILITY AND SETUP}

The twin-channel hot film anemometer model 1050 and the $\mathrm{X}$ shape hot film probe model 1243 imported from the United States were used. Two hot film probes were calibrated in the hot film probe calibrator model 10120 (as Figure 1) before measuring, the results are shown as Figure 2 and Figure 3. The HWDAP software system was adopted to process the data.

An open circulating water channel (as Figure 4) made of organic glass was used to carry out the experiments. The length, width and depth of the experimental section was respectively $1.40 \mathrm{~m}, 0.14 \mathrm{~m}$ and $0.15 \mathrm{~m}$. For the experimental section flow, the background turbulence intensity was less than $3.5 \%$, flow field uniformity was $0.6 \%$, velocity could adjust continuously from zero to $0.4 \mathrm{~m} / \mathrm{s}$. Measuring point started from the place $0.7 \mathrm{~m}$ far from the entrance, three group data was obtained through measuring at three sections. In the measuring, the sampling frequency was $1 \mathrm{kHz}$, the sample time 4s, and the Reynolds number (ReH) 43600.

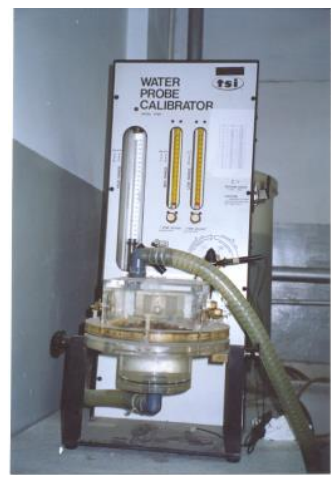

Figure 1. Photo of hot film probe calibrator 


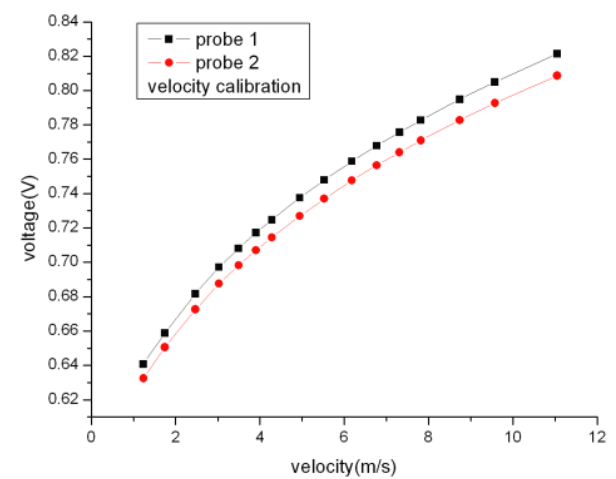

Figure 2. Velocity Calibration curve of X-shape hot film probe

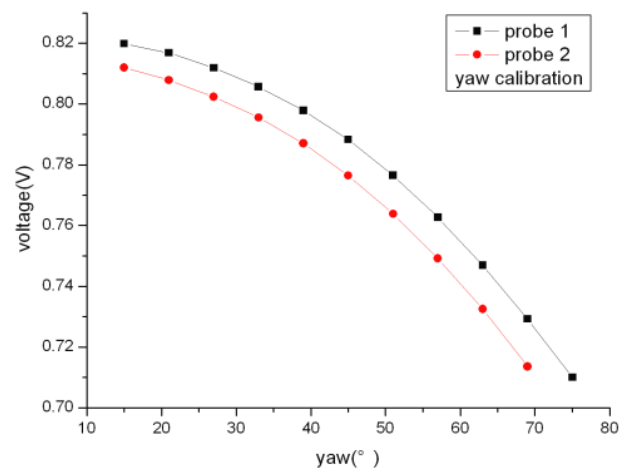

Figure 3. the yaw Calibration curve of X-shape hot film probe

\section{EXPERIMENTAL RESULTS AND DISCUSSION}

\subsection{The measurement of the mean flow field}

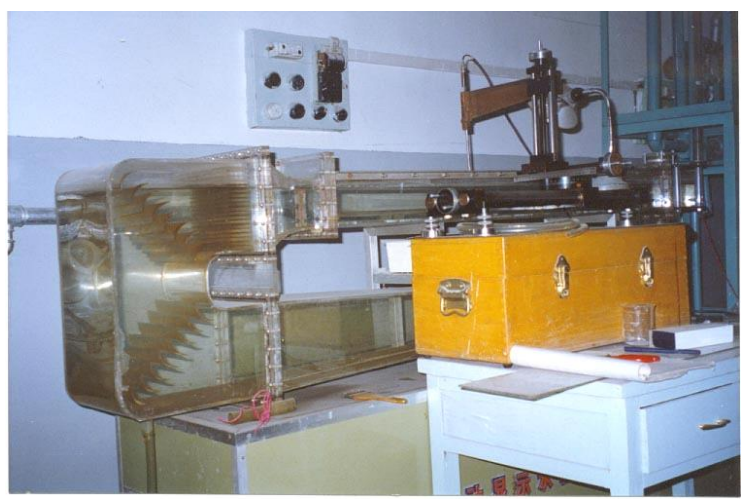

Figure 4. Photo of open circulating water channel

The mean velocity profile in the near wall region was measured using hot film probe. Mean velocity profile of turbulent flow in the semi-section of water channel under the dimensionless of scale is shown as Figure 5. In Figure $5, u^{+}=u / u^{*}, y^{+}=y u^{*} / v, u^{+}$is dimensionless velocity, $u^{*}$ is friction velocity, $y^{+}$is dimensionless distance from the wall, $v$ is dynamic viscosity of water under normal temperature. The results show that the curve is consistent with logarithmic law very well.

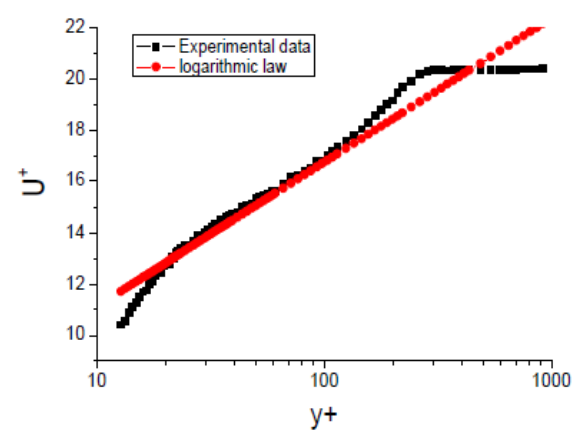

Figure 5. Mean velocity profile of turbulent flow $\left(\mathrm{Re}_{\mathrm{H}}=43600, \mathrm{f}=1 \mathrm{khz}, \mathrm{t}=4 \mathrm{~s}\right)$

\subsection{Distribution of Reynolds stress in the boundary layer}

The distribution of three Reynolds stresses in wall-normal direction was obtained by statistic calculations of the collected data along the whole channel. The relations between Reynolds stresses and $y^{+}$are ploted with Reynolds stresses as ordinate against $y^{+}$as abscissa (as Figure 6). The results in the figure indicate as follow: the value of Reynolds stresses reach maximum at the wall, then gradually decrease with $y^{+}$, the value of $\mathrm{u}^{\prime 2}$ is maximum while the value of $u^{\prime} v^{\prime}$ and $v^{\prime 2}$ is close to. Those results agree with the classical results by Klebanoff[12] ( as Figure 7) and Hinze[8] and show that our Measuring could reflect correctly the distributions of flow field.

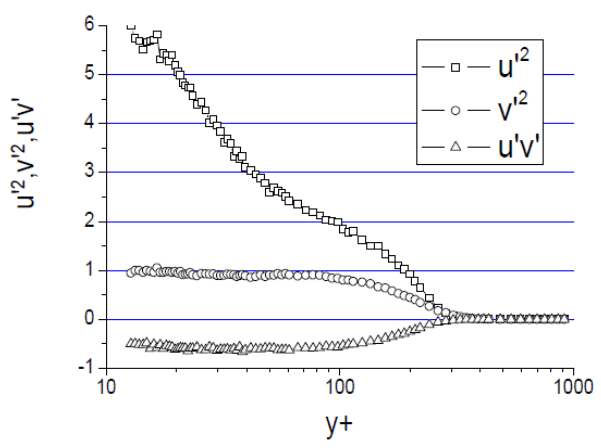

Figure 6. Reynolds stress distribution in wall-normal direction $\left(\mathrm{Re}_{\mathrm{H}}=43600, \mathrm{f}=1 \mathrm{khz}, \mathrm{t}=4 \mathrm{~s}\right)$

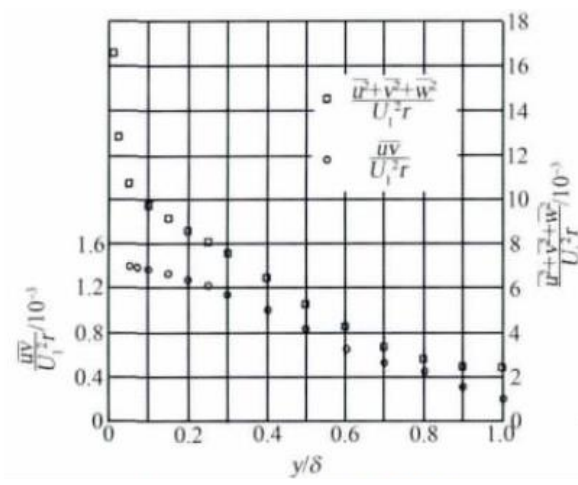

Figure 7. Reynolds stress distribution in wall-normal direction by Klebanoff 


\subsection{Spatial distribution laws for each components of eddy viscosity tensor}

For two-dimensional channel turbulence flow, the components of Reynolds stress include $-\rho \overline{u^{\prime 2}},-\rho \overline{v^{\prime 2}}$ and $-\rho \overline{u^{\prime} v^{\prime}}$, the components of mean strain rate include only $\partial \bar{u} / \partial y$, so the non-zero components of the eddy viscosity tensor are $\varepsilon_{1112}, \varepsilon_{2212}$ and $\varepsilon_{1212}$. And, the formula (8) (10) are obtained by formula (7):

$$
\begin{aligned}
& \varepsilon_{1112}=\varepsilon_{1121}=\left(2 k / 3-\rho \overline{u^{\prime} u^{\prime}}\right) /(\partial \bar{u} / \partial y) \\
& \varepsilon_{2212}=\varepsilon_{2221}=\left(2 k / 3-\rho \overline{v^{\prime} v^{\prime}}\right) /(\partial \bar{u} / \partial y) \\
& \varepsilon_{1212}=\varepsilon_{1221}=\rho \overline{u^{\prime} v^{\prime}} /(\partial \bar{u} / \partial y)
\end{aligned}
$$

The profiles of $\varepsilon_{1112}, \varepsilon_{2212}$ and $\varepsilon_{1212}$ are drawn respectively in Fig 8 Fig10. The abscissa nondimensionalize with value of $y^{+}$, and the vertical coordinates nondimensionalize with the value of $u_{*}^{2} / v$.The mean velocity gradient $\partial \bar{u} / \partial y$ is calculated using the finite difference method.

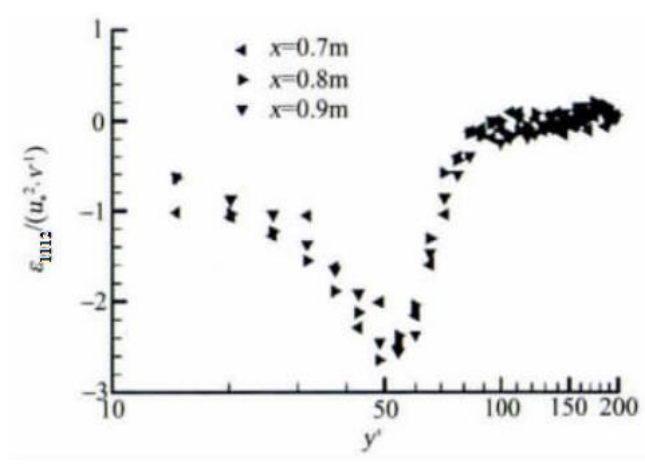

Figure 8. ${ }^{\varepsilon_{1112}}$ profile in wall-normal direction $(\mathrm{ReH}=43600$, $\mathrm{f}=1 \mathrm{khz}, \mathrm{t}=4 \mathrm{~s})$

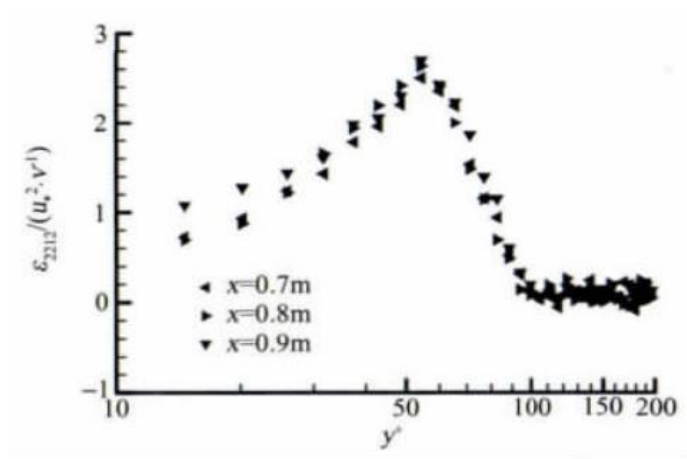

Figure 9. ${ }^{\varepsilon_{2212}}$ profile in wall-normal direction $(\mathrm{ReH}=43600$, $\mathrm{f}=1 \mathrm{khz}, \mathrm{t}=4 \mathrm{~s})$

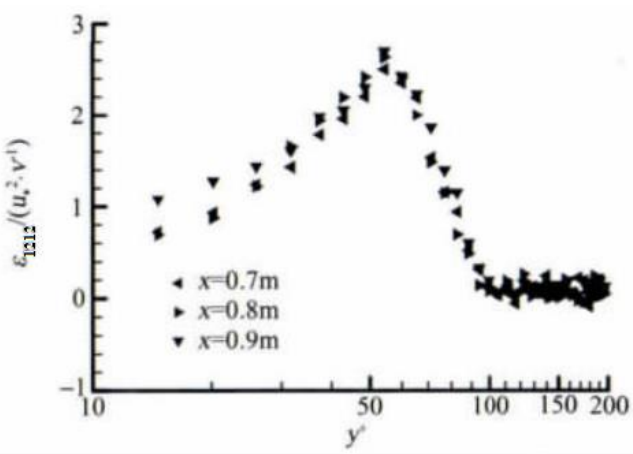

Figure 10. ${ }^{\varepsilon_{1212}}$ profile in wall-normal direction $(\mathrm{ReH}=43600, \mathrm{f}=1 \mathrm{khz}, \mathrm{t}=4 \mathrm{~s})$

If we use Boussinesq hypothesis equation (1), the values of normal Reynolds stress are zero, it does not reflect the contributions of normal Reynolds stress $-\rho \overline{u^{\prime 2}},-\rho \overline{v^{\prime 2}}$ to turbulence flow.But the experimental results above indicate that the values of normal Reynolds stress $-\rho \overline{u^{\prime 2}},-\rho \overline{v^{\prime 2}}$ are related with the mean velocity gradient and may influence turbulence flow. It shows that the tensorial eddy viscosity model will be more reasonable than the model of Boussinesq hypothesis.

As can be seen from the figures, the values of three components of eddy viscosity tensor have the same changing trend, reach the maximum at the point $y^{+}=55$ about, and then gradually falls to zero. The value of $\varepsilon_{1112}$ is negative which demonstrate the contribution of $\partial \bar{u} / \partial y$ to $-\rho \overline{u^{\prime 2}}$ is negative. This results agree with the theory of counter gradient transport in turbulent flows[13 15]. Otherwise, the values of $\varepsilon_{2212}$ and $\varepsilon_{1212}$ are positive which demonstrate the contribution of $\partial \bar{u} / \partial y$ to $-\rho \overline{v^{\prime 2}}$ and $-\rho \overline{u^{\prime} v^{\prime}}$ are positive. The profile of $\varepsilon_{1212}$ agrees with the experimental result of Klebanoff [12], Townsend [16] and Sivakumar[17] in the turbulent boundary layer .

\section{CONCLUSION}

The tensorial eddy viscosity turbulence model is investigated through experiments by using hot film anemometer and water probe in a water channel The results show that the Renolds stress distributions keep consistent with the classic results of the turbulent. The normal profiles of each component of eddy viscosity tensor are discussed. The results of eddy viscosity tensor indicate that the tensorial eddy viscosity turbulence model contains the influence of normal Reynolds stress which reflect the anisotropy of turbulence. The anisotropic turbulence model is extremely important because almost all turbulence flow are anisotropic in engineering. The anisotropic tensorial eddy viscosity model can reflect the physical nature of Reynolds stress. This investigation provides an experimental evidence for future study of the model prediction . 


\section{ACKNOWLEDGMENT}

The authors would like to thank the chinese National Science Foundation(10832001) and the Doctor Scientific Foundation of Henan Polytechnic University (72515/413).

\section{REFERENCE}

[1] N. Oumrani, M. Aouissi, A. Bounif, B. Yssaad, et al., "A first -and second-order turbulence models in hydrogen non-premixed flame," International Journal of Heat and Technology, vol. 33, no. 3, pp. 27-34, 2015. DOI: $10.18280 /$ ijht.330304.

[2] Sanjay K. Sharman and Vilas R. Kalamkar, "Computational fluid dynamics approach in thermohydraulic analysis of flow in ducts with rib roughened walls - A review," Renewable and Sustainable Energy Review, vol. 55, no. 3, pp. 756-788, 2016.

[3] Boussinesq J., "Theorie de l'écoulement tourbillant," Mém. Prés. Pardiv. Savant a Lacad. Sci., Paris, vol. 23, pp. 46-50, 1877.

[4] Tomás Chacón Rebollo, Mathematical and Numerical Foundations of Turbulence Models and Applications, London: Springer New York Heidelberg Dordrecht, 2014.

[5] Stephen B. Pope, Turbulent Flow, United Kingdom: Cambridge University Press, 2000.

[6] Peter R. Lang and Frank S. Lombargo, Atmospheric Turbulence, Meteorological Modeling and Aerodynamics, New York: Nova Science Publishers, 2010.

[7] Tennekes, H. and Lumley, J. L., A First Course in Turbulence, Cambridge: MIT Press, 1983.

[8] J. O. Hinze, Turbulence, 2nd edition, New York: Mcgraw-Hill Book Company, 1975.

[9] F. Corvaro, G. Nardini, M. Paroncini and R. Vitali, "PIV and numerical analysis of natural convective heat transfer and fluid flow in a square cavity with two vertical obstacle," International Journal of Heat and Technology, vol. 33, no. 2, pp. 51- 55, 2015. DOI: 10.18280/ijht.330208.

[10] Wang Zhendong and Jiang Nan, "Study of new eddy viscosity modelling for turbulence," Chinese Science Bulletin, vol. 39, no. 19, pp. 1593-1597, 1994.

[11] Wang Zhendong and Wu Shuangqun, "New Eddy viscosity modelling of turbulent boundary layer with adverse pressure radient," Chinese Science Bulletin, vol. 41, no. 2, pp. 157-162, 1996.

[12] Klebanoff, P. S. "Characteristics of turbulence in a boundary-layer with zero pressure gradient," NACA Technical Note 3178, 1954; also NACA Report 1247, 1955.

[13] Qiu Xiang, Jiang Jianbo and Liu Yulu, "Effects of pressure-gradient on turbulent counter-gradient transport," Chinese Journal of Theoretical and Applied Mechanics, vol. 36, no. 2, pp. 163-170, 2004.

[14] Veynante D. and Poinsot T., "Efects of pressure gradient on turbulent premixed flame," J. Fluid Mech., vol. 353, pp. 83-114, 1997. DOI: $10.1017 / \mathrm{S} 0022112097007556$.

[15] Sommer T. P., "Nonequilibrium and history effects of homogeneous turbulence in a stably stratified medium," Int. J Heat and Fluid Flow, vol. 18, no. 1, pp. 29-37, 1997. DOI: $\underline{10.1016 / S 0142-}$ 727X(96)00140-3.

[16] Townsend A. A., "The structure of the turbulent boundary layer," Mathematical Proceedings of the Cambridge Philosophical Society, vol. 47, no. 2, pp. 375-395, 1951.

[17] K. Sivakumar, E. Natarajan and N. Kulasekharan, "Experimental studies on turbulent flow in ribbed rectangular convergent ducts with different rib sizes," International Journal of Heat and Technology, vol. 32, no. 1, pp. 79-85, 2014 